\title{
ON A PROBLEM OF ERDÖS ON SEQUENCES AND MEASURABLE SETS
}

\author{
K. J. FALCONER
}

\begin{abstract}
Erdös has conjectured that given a decreasing sequence of real numbers convergent to 0 there always exists a measurable set of positive measure that contains no similar copy of the sequence. We prove this conjecture if the sequence does not converge too rapidly.
\end{abstract}

A long standing conjecture of Erdös (see for example, [ 3 or 4 ]) is that given any decreasing sequence $\left\{x_{n}\right\}_{n=1}^{\infty}$ of real numbers with $x_{n} \rightarrow 0$, there exists a Lebesgue measurable set $E$ of positive Lebesgue measure containing no similar copy of the sequence.

Miller [5] and Arias de Reyna [1] (see also the references contained in these papers) have obtained various results related to this conjecture but with (nonmeasurable) sets of second Baire category instead of measurable sets of positive Lebesgue measure. Borwein and Ditor [2] investigate the problem with similarity replaced by congruence.

Here we give a partial proof of the conjecture on the assumption that the sequence converges 'slower than geometrically'. We denote Lebesgue measure on the line by $m$.

THEOREM. Let $\left\{x_{n}\right\}_{n=1}^{\infty}$ be a decreasing sequence of real numbers convergent to 0 such that

$$
\lim _{n \rightarrow \infty} \frac{x_{n+1}}{x_{n}}=1 .
$$

Then there exists a closed set $E$ with $m(E)>0$ such that for any numbers $b, c$ with $c \neq 0, c x_{n}-b \notin E$ for infinitely many $n$.

Proof. Choose numbers $\lambda_{k}(1 \leqslant k<\infty)$ such that $0<\lambda_{k}<1$ and $\sum_{k=1}^{\infty} \lambda_{k}<\frac{1}{2}$. For a rapidly decreasing sequence of positive lengths $\left\{l_{k}\right\}$ (to be specified later) let

$$
E_{k}=\bigcup_{r=-\infty}^{\infty}\left[r l_{k}, r l_{k}+l_{k}\left(1-\lambda_{k}\right)\right]
$$

and let $E=\cap_{k=1}^{\infty} E_{k}$. Then $E$ is closed, and provided that $l_{k}<1(1 \leqslant k<\infty)$, $m(E)>0$, since

$$
m([0,1] \backslash E) \leqslant \sum_{k=1}^{\infty} m\left([0,1] \backslash E_{k}\right) \leqslant \sum_{k=1}^{\infty} \lambda_{k} l_{k}\left(\frac{1}{l_{k}}+1\right) \leqslant \sum_{k=1}^{\infty} 2 \lambda_{k}<1 .
$$

Received by the editors February 20, 1983.

1980 Mathematics Subject Classification. Primary 28A75. 
For each $k$ we may, using (1), find $n(k)$ (increasing with $k$ ) such that if $n \geqslant n(k)$ then

$$
1-\frac{1}{2} \lambda_{k}<\frac{x_{n+1}}{x_{n}}
$$

so that

$$
\frac{1}{x_{n+1}} l_{k}\left(2-\lambda_{k}\right)<\frac{1}{x_{n}} 2 l_{k}
$$

Thus if $0 \leqslant b \leqslant l_{k}$,

$$
\frac{1}{x_{n+1}}\left(l_{k}\left(1-\lambda_{k}\right)+b\right)<\frac{1}{x_{n}}\left(l_{k}+b\right) \text {. }
$$

Hence for each $n \geqslant n(k)$, the intervals

$$
\frac{1}{x_{n}}\left(l_{k}\left(1-\lambda_{k}\right)+b, l_{k}+b\right) \text { and } \frac{1}{x_{n+1}}\left(l_{k}\left(1-\lambda_{k}\right)+b, l_{k}+b\right)
$$

overlap. We conclude that the interval $\left(\left(l_{k}\left(1-\lambda_{k}\right)+b\right) / x_{n(k)}, \infty\right)$ lies in the complement of $\cap_{n=n(k)}^{\infty}\left(E_{k}+b\right) / x_{n}$. Together with a similar argument for the negative numbers this implies

$$
\bigcap_{n=n(k)}^{\infty} \frac{1}{x_{n}}\left(E_{k}+b\right) \subseteq\left[-2 l_{k} / x_{n(k)}, 2 l_{k} / x_{n(k)}\right]
$$

if $0 \leqslant b \leqslant l_{k}$, and so, by the periodicity of $E_{k}$, for all real $b$. Now for any integer $m$

$$
\begin{aligned}
\bigcap_{n=n(m)}^{\infty} \frac{1}{x_{n}}(E+b) & =\bigcap_{n=n(m)}^{\infty} \frac{1}{x_{n}}\left(\bigcap_{k=1}^{\infty} E_{k}+b\right) \subseteq \bigcap_{n=n(m)}^{\infty} \frac{1}{x_{n}}\left(\bigcap_{k=m}^{\infty} E_{k}+b\right) \\
& =\bigcap_{k=m}^{\infty} \bigcap_{n=n(m)}^{\infty} \frac{1}{x_{n}}\left(E_{k}+b\right) \subseteq \bigcap_{k=m}^{\infty} \bigcap_{n=n(k)}^{\infty} \frac{1}{x_{n}}\left(E_{k}+b\right) \\
& \subseteq \bigcap_{k=m}^{\infty}\left[-2 l_{k} / x_{n(k)}, 2 l_{k} / x_{n(k)}\right]=\{0\}
\end{aligned}
$$

provided that the numbers $l_{k}$ are chosen so that $l_{k} / x_{n(k)} \rightarrow 0$ as $k \rightarrow \infty$. Thus for any $c \neq 0, c \notin(E+b) / x_{n}$ or $c x_{n} \notin E+b$ for some $n \geqslant n(m)$, completing the proof.

\section{REFERENCES}

1. J. Arias de Reyna, Some results connected with a problem of Erdös. III, Proc. Amer. Math. Soc. 89 (1983), 291-292.

2. D. Borwein and S. Z. Ditor, Translates of sequences in sets of positive measure, Canad. Math. Bull. 21 (1978), 497-498.

3. P. Erdös, Problems, Math. Balkanica 4 (1974), 203-204.

4. __ My' Scottish Book 'problems', The Scottish Book (R. D. Mauldin, Ed.), Birkhäuser, Boston, Mass., 1981, pp. 3j-43.

5. H. I. Miller. Some results connected with a problem of Erdös. II. Proc. Amer. Math. Soc. 75 (1979). $265-268$.

School of Mathematics, University Walk. Bristol, BS8 1TW. England 